\title{
Human fetal mesenchymal stem cells differentiate into brown and white adipocytes: a role for ERR $\alpha$ in human UCP1 expression
}

\author{
Daniel L Morganstein ${ }^{1}$, Pensee $\mathrm{Wu}^{2}$, Meritxell R Mane ${ }^{1}$, Nick M Fisk ${ }^{2}$, Roger White ${ }^{1}$, Malcolm G Parker ${ }^{1}$ \\ ${ }^{I}$ Department of Molecular Endocrinology, Institution of Reproductive and Developmental Biology, Imperial College London, Lon- \\ don, UK; ${ }^{2}$ Department of Experimental Fetal Medicine, Institution of Reproductive and Developmental Biology, Imperial College \\ London, London, UK
}

We investigated the ability of fetal mesenchymal stem cells (fMSCs) to differentiate into brown and white adipocytes and compared the expression of a number of marker genes and key regulatory factors. We showed that the expression of key adipocyte regulators and markers during differentiation is similar to that in other human and murine adipocyte models, including induction of PPAR $\gamma 2$ and FABP4. Notably, we found that the preadipocyte marker, Pref-1, is induced early in differentiation and then declines markedly as the process continues, suggesting that fMSCs first acquire preadipocyte characteristics as they commit to the adipogenic lineage, prior to their differentiation into mature adipocytes. After adipogenic induction, some stem cell isolates differentiated into cells resembling brown adipocytes and others into white adipocytes. Detailed investigation of one isolate showed that the novel brown fat-determining factor PRDM16 is expressed both before and after differentiation. Importantly, these cells exhibited elevated basal UCP-1 expression, which was dependent on the activity of the orphan nuclear receptor ERRa, highlighting a novel role for ERRa in human brown fat. Thus fMSCs represent a useful in vitro model for human adipogenesis, and provide opportunities to study the stages prior to commitment to the adipocyte lineage. They also offer invaluable insights into the characteristics of human brown fat.

Keywords: mesenchymal stem cells, adipogenesis, adipocytes, brown, prdm16, ucp1, ERR $\alpha$

Cell Research (2010) 20: 434-444. doi: 10.1038/cr.2010.11; published online 26 January 2010

\section{Introduction}

The developmental programmes that control the generation of white adipose as a storage depot for triglyceride versus brown fat for adaptive thermogenesis are poorly understood. While they both express common marker genes including fatty acid-binding protein 4 (the human homolog of aP2 in the mouse), brown fat has a higher mitochondrial content and increased expression of many genes involved in adaptive thermogenesis [1]. The regulation of adipogenesis from preadipocytes has been examined extensively in murine models [2] and led

Correspondence: Malcolm G Parker

Tel:+ 44207594 2177; Fax: + 442075942184

E-mail:m.parker@imperial.ac.uk

Received 25 June 2009; revised 3 November 2009; accepted 8 December 2009; published online 26 January 2010 to the identification of PPAR $\gamma$ and $\mathrm{C} / \mathrm{EBP} \alpha$ as key transcription factors. Conversely, Pref-1 (preadipocyte factor 1, also identified as Dlk1), a trans-membrane protein related to the EGF (epidermal growth factor) that is highly expressed in preadipocytes, blocks adipogenesis but its levels fall with the onset of differentiation. Exogenous expression of Pref-1 blocks differentiation of 3T3-L1 preadipocytes [3], while its overexpression in transgenic mice results in lipodystrophy [4]; Pref-1 null mice on the other hand have accelerated adiposity [5].

It was generally assumed that brown and white fat cells were derived from a common adipogenic progenitor. However, expression profiling indicated that brown fat cells express a large number of muscle-related genes [6], and genetic studies suggest that brown adipose tissue may have a distinct origin from that of brown adipocytes found in white adipose tissue [6]. Moreover, a population of cells derived from human skeletal muscle can differ- 
entiate in vitro into brown adipocytes [7]. Recent work indicates that brown adipose tissue and skeletal muscle are derived from common Myf5-expressing progenitor cells and that the transcriptional regulator, PRDM16, controls a brown fat/skeletal muscle switch [8]. Exogenous PRDM16 expression was able to drive brown adipogenesis, but only if expressed prior to the onset of differentiation [9]. Nevertheless, brown fat cells that appear in white adipose tissue on cold exposure do not appear to be derived from these Myf5-expressing progenitor cells [8], and so it is conceivable that these brown fat cells are derived from a common adipogenic precursor cell.

Interestingly, manipulation of a number of cofactors for nuclear receptors suggests that it may be possible to switch white fat into brown fat. Thus, exogenous expression of PGC1 $\alpha[10]$ in white fat cells or the deletion of the corepressor RIP140 results in the expression of UCP1 [11-13], a characteristic marker of brown fat cells. The coactivator PGC1 $\beta$ is also important for the expression of many typical brown fat genes [14].

Mesenchymal stem cells can be expanded in vitro and induced to differentiate into cells of multiple mesenchymal lineages [15]. Human adult mesenchymal stem cells have been shown to differentiate into white adipocytes [16], but their ability to differentiate into brown adipocytes is unclear. One study of human amniotic fluidderived stem cells did address the phenotype of the differentiated cells and found low levels of expression of the brown fat markers UCP-1 and PGC1 $\alpha$. However, this expression was lower than that seen in adult sub-cutaneous white adipocytes [17], which the differentiated stem cells most closely resembled. Mesenchymal stem cells have been isolated from blood, liver and bone marrow of first-trimester human fetuses, and these can also differentiate into adipocytes [18, 19]. However, the process of adipogenesis and the functional properties of the derived adipocytes were only minimally characterized. Therefore, we have studied first-trimester cells to investigate their potential to differentiate into either white or brown adipocytes. We also studied the process of adipogenesis in fetal mesenchymal stem cells (fMSCs) to determine whether key markers and regulators were expressed in a similar manner to that previously described in other adipocyte models $[2,13,20,21]$, and to characterize further the differentiated adipocytes.

\section{Results}

\section{Adipogenic differentiation of fMSCs}

Initially, we confirmed previous reports that fMSCs were able to differentiate into adipocytes [18]. After 14 days, droplets were clearly visible in $\sim 30 \%$ of cells, and staining with Oil Red O confirmed the presence of intracellular lipid (Figure 1A). To establish the mesenchymal stem cell origin of the isolates, we demonstrated that they were also able to differentiate into bone (Figure 1A). We next analyzed the expression of a number of adipocyte markers and regulatory factors that were expressed during mouse adipogenesis. Expression analysis was performed with two different isolates (one from fetal blood and one from fetal bone marrow from different fetuses) before and after treatment with adipogenic differentiation media. Expression of the adipocyte marker fatty acid-binding protein 4 (the human homolog of the mouse gene aP2) was detected after 3 days, and increased progressively with a 150 -fold induction after 14 days (Figure 1B), in parallel with lipid accumulation.

The key adipogenic regulator PPAR $\gamma$ was expressed in undifferentiated stem cells, but increased throughout differentiation, and after 14 days showed an 8- to 12 -fold upregulation compared to untreated cells. PPAR $\gamma 2$, the adipocyte-specific isoform of PPAR $\gamma$ was not expressed in undifferentiated cells, but was induced markedly during differentiation (Figure 1B). Expression of $\mathrm{C} / \mathrm{EBP} \alpha$ also increased following differentiation (Figure 1B). Pref-1/Dlk1, which suppresses the differentiation of mouse preadipocytes into adipocytes, was not detectable in confluent untreated fMSCs, but addition of adipogenic cocktail led to expression within $6 \mathrm{~h}$, consistent with the appearance of preadipocytes (Figure 1B). Expression levels increased for 7 days, but declined markedly by day 14 , when a significant proportion of preadipocytes had undergone differentiation into mature adipocytes. It seems, therefore, that fMSCs express many markers and regulators of adipocytes at the appropriate times and the pattern of expression of these genes was remarkably similar in the two isolates of fMSCs examined.

\section{Isolates of fMSCs can differentiate into either brown or white adipocytes}

The ability of fMSCs to differentiate into brown fat cells was examined by determining the expression of UCP-1 after adipogenic differentiation using identical differentiation conditions shown to promote adipogenesis in adult MSCs (adapted from Janderova et al. [22]). Analysis of five different isolates indicated a wide range of UCP-1 expression (Supplementary information, Figure $\mathrm{S} 1$ ), and so we examined two from fetal bone marrow in detail. One obtained at 10 weeks plus 1-day gestation (BM $10^{+1}$ ) gave rise to adipocytes with virtually undetectable expression of UCP1, while the other from a 9 weeks and 4-day gestation fetus $\left(\mathrm{BM} \mathrm{9}^{+4}\right)$ differentiated into adipocytes that expressed high levels of UCP1 (Figure $2 \mathrm{~A})$. The mesenchymal stem cell identity of both isolates 
A

(1) Undifferentiated

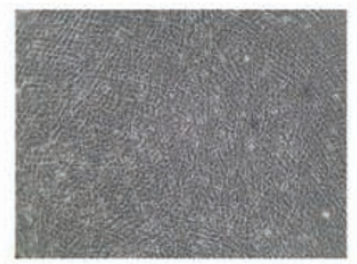

(4) Bone differentiation

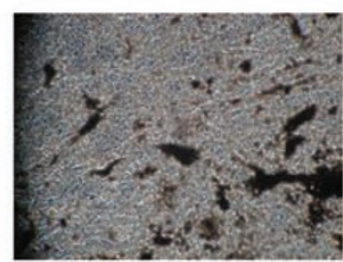

B
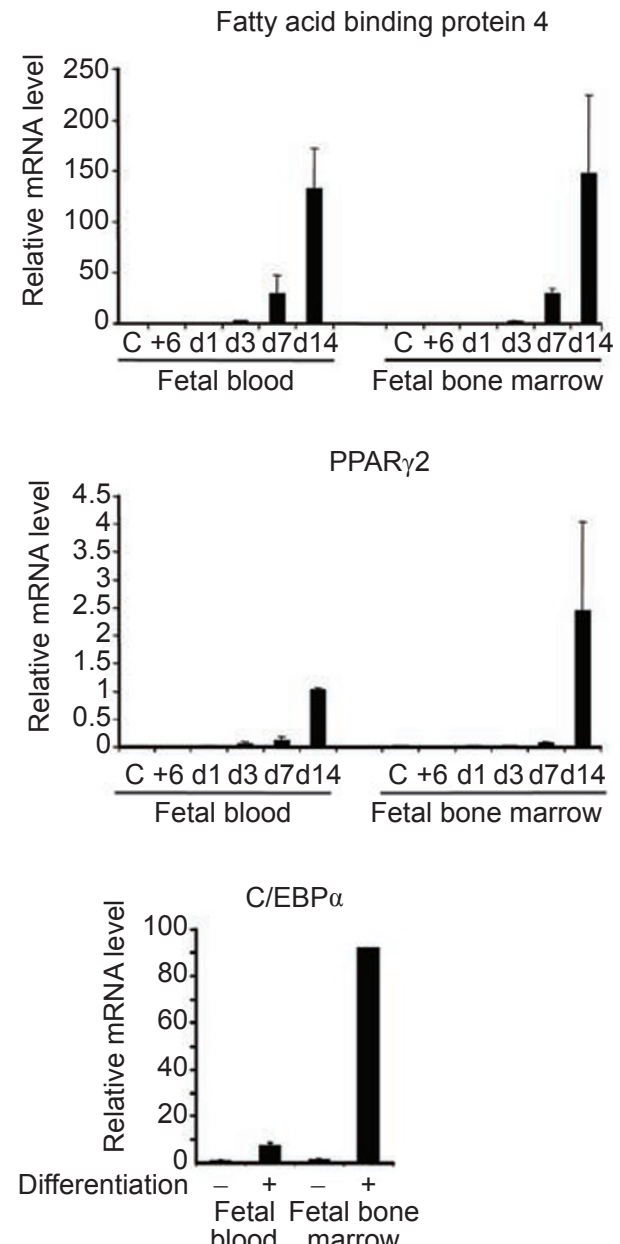

(2) Adipogenic differentiation

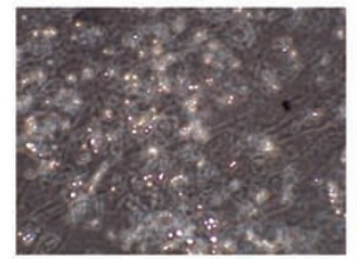

(3) Oil Red O stain

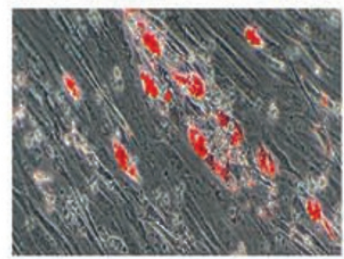

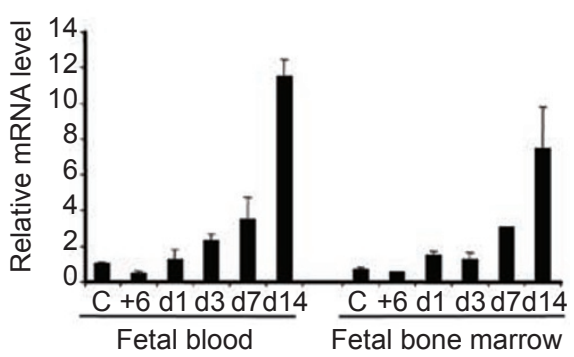

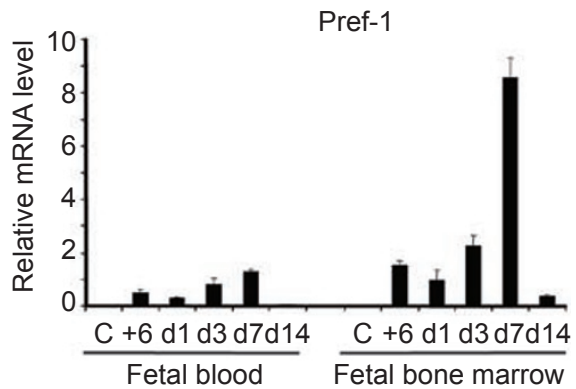

Figure 1 Fetal mesenchymal stem cells differentiate into adipocytes. (A) Fetal mesenchymal stem cells before (1) and after differentiation into adipocytes and bone, as demonstrated by light microscopy (2), Oil Red O staining to demonstrate lipid accumulation (3) or Von Kossa staining to demonstrate mineralization (4) ( $\times 400$ magnification). (B) Time course of mRNA expression of key markers and regulators of adipocytes by quantitative PCR. C, confluent cells, +6, $6 \mathrm{~h}$ post hormonal induction of differentiation, all other time points indicate days post induction. Fetal blood and fetal bone marrow indicate the source of isolate. Error bars indicate SD. Relative expression in arbitrary units. 
A

UCP-1

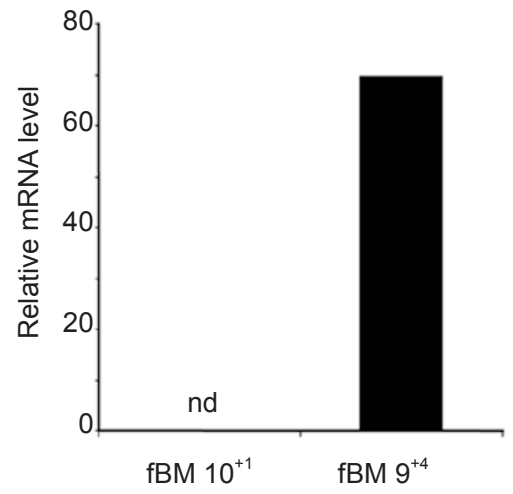

C

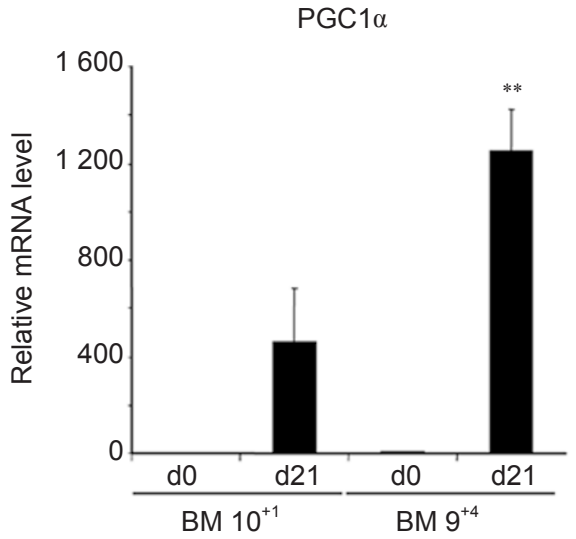

D

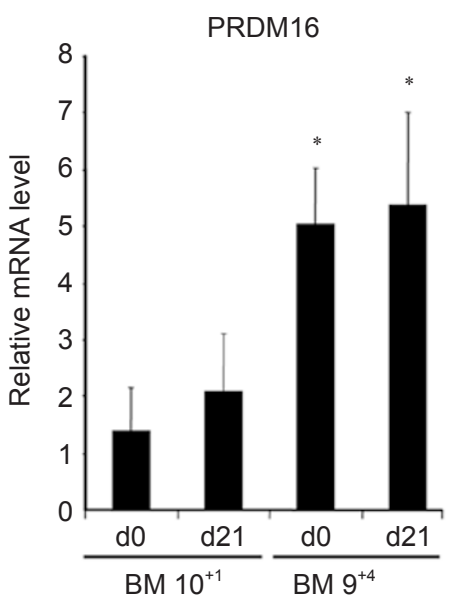

B

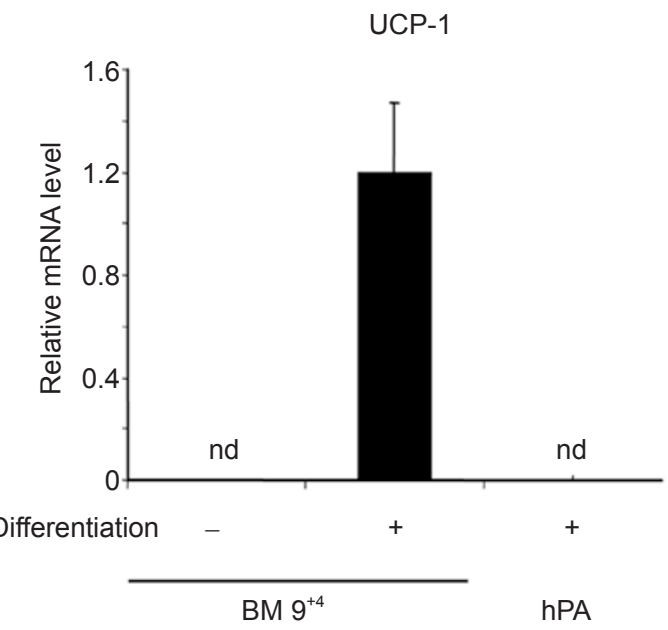

RIP140

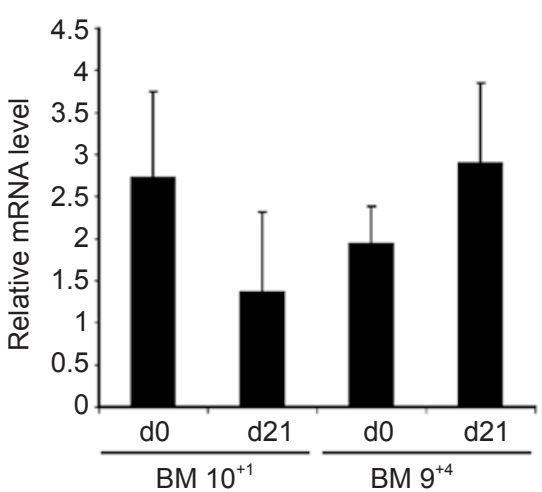

$E$

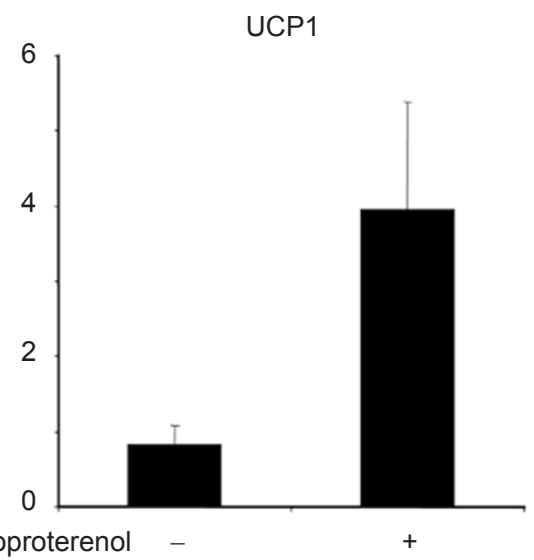

Figure 2 Two different isolates of fetal mesenchymal stem cells differentiate into adipocytes that resemble white and brown adipocytes, respectively. (A) UCP-1 mRNA expression in $\mathrm{fMSC} 10^{+1}$ and $9^{+4}$ isolates after 21 days of differentiation. (B) UCP1 mRNA expression in $\mathrm{fMSC} 9^{+4}$ isolates before and after 21 days of differentiation, and differentiated human adult sub-cutaneous adipocytes. (C and D) mRNA expression of PGC1 $\alpha$ and RIP140 (C) and PRDM16 (D) in the two isolates before and after differentiation. ** $P<0.001, * P<0.05$ for comparison between the two isolates. (E) Expression of UCP-1 mRNA in $9^{+4}$ isolate after 21 days differentiation, before and after treatment with $10 \mu \mathrm{M}$ isoproteranol for $5 \mathrm{~h}$. Error bars indicate SD and nd indicates not detected. Relative expression was in arbitrary units. 
was confirmed by characteristic expression of markers, $\mathrm{SH} 2, \mathrm{SH} 3$ and vimentin positive, and CD14, 34 and 45 negative (Figure 3 ), and by their ability to differentiate into bone (data not shown). Expression of UCP1 was dependent on differentiation, and was considerably higher than the levels of UCP1 found in human adult white adipocytes derived from the in vitro differentiation of subcutaneous preadipocytes (Figure 2B). Given that UCP1 gene expression is induced during thermogenesis in response to PGC1 $\alpha$ expression [10], we analyzed the ex- pression of this co-factor. PGC1 $\alpha$ expression was markedly induced in both isolates following differentiation, and so whilst it is expressed at higher levels in the BM 9 -week isolate compared to the 10 -week cells, it cannot account for the marked difference in UCP1 gene expression. The possibility that differential RIP140 expression might account for the difference in UCP1 expression was also investigated, but its level was similar in both isolates before and after differentiation (Figure 2C). The brown fat regulator PRDM16 was also expressed in both
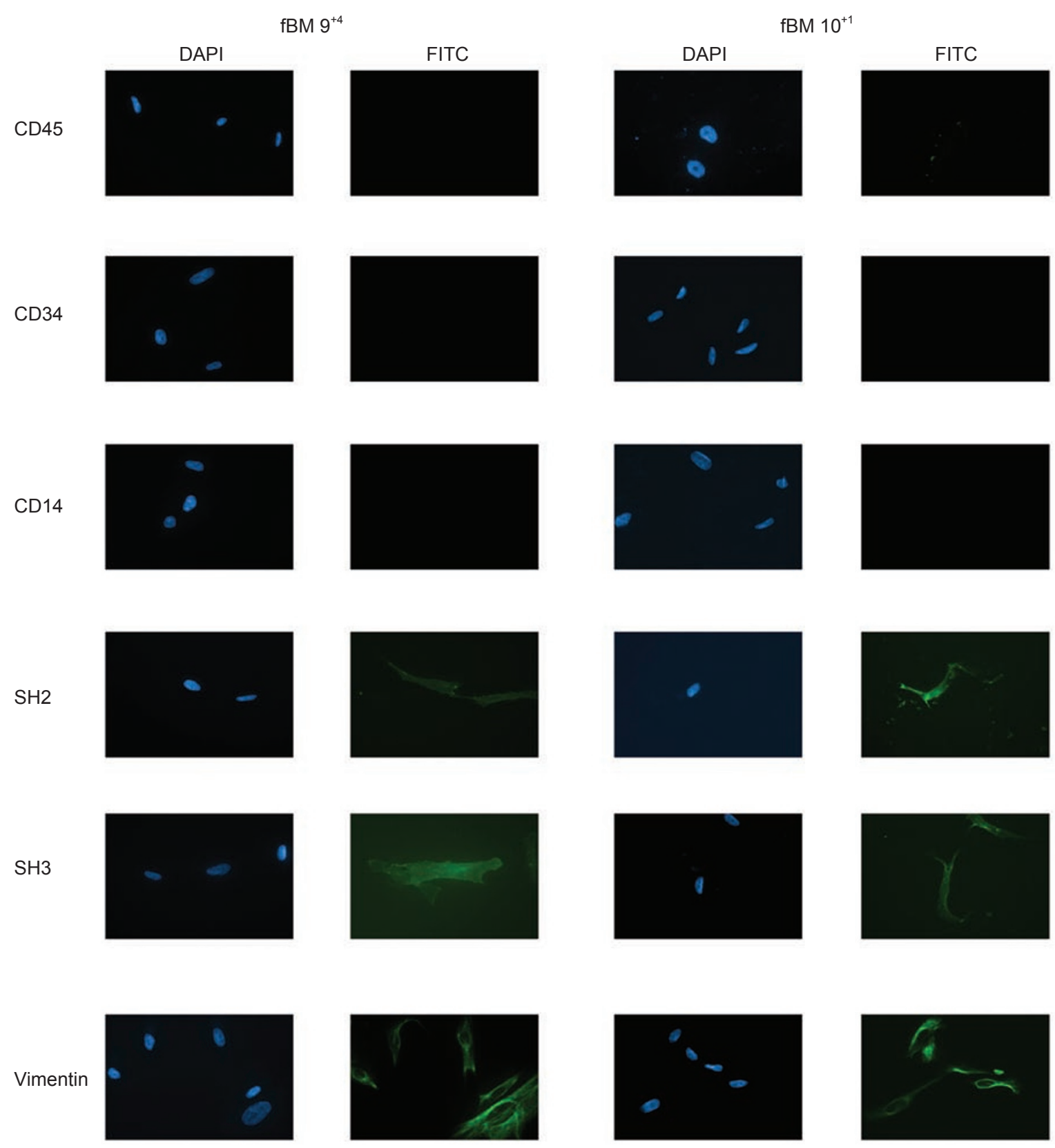

Figure 3 Immunophenotyping confirms mesenchymal stem cell characterisitics. Undifferentiated $9^{+4}$ and $10^{+1} \mathrm{fMSCs}$ express $\mathrm{SH} 2, \mathrm{SH} 3$ and vimentin, but not CD14, CD 34 or CD45, confirming their mesenchyme origin. 
A

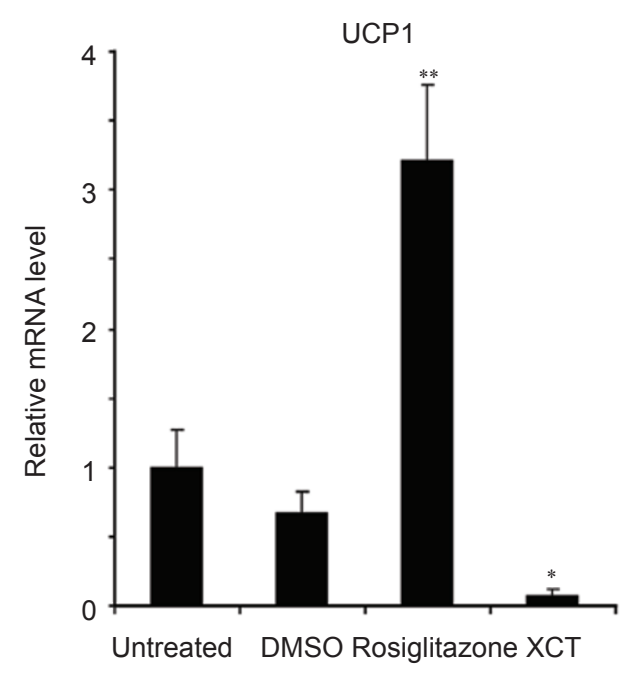

C

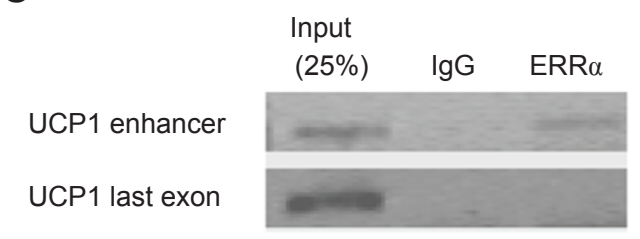

E

ERR $\alpha$
B
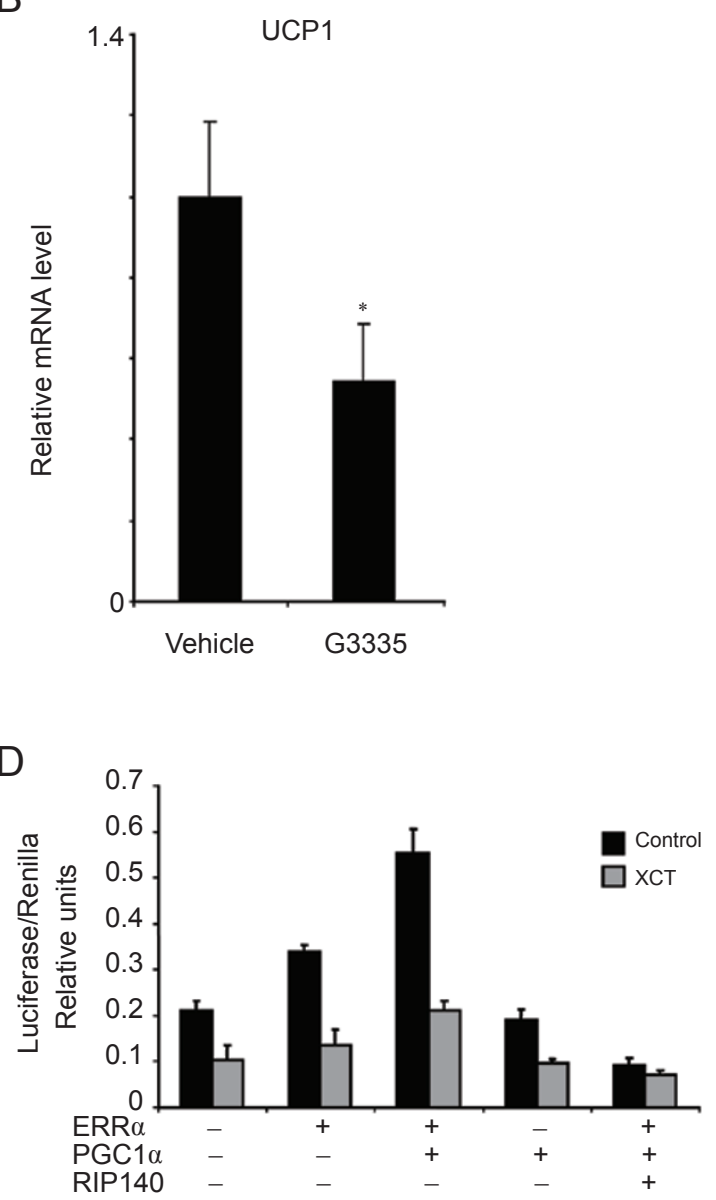

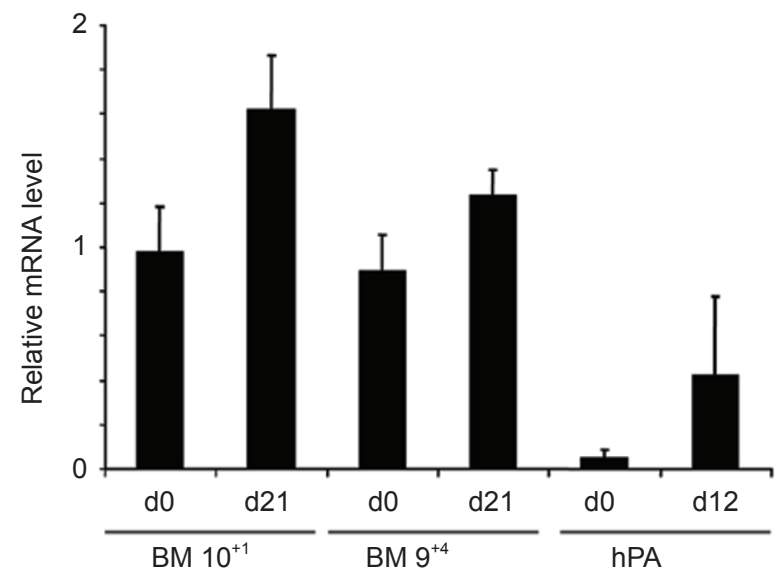

Figure 4 UCP-1 expression in fMSC $9^{+4}$ adipocytes can be induced by Rosiglitazone treatment, and reduced by the ERR $\alpha$ inverse agonist. (A) UCP-1 expression following Rosiglitazone or XCT790 treatment for $48 \mathrm{~h} . * P<0.05, * * P<0.005$. (B) UCP-1 expression after $48 \mathrm{~h}$ treatment with PPAR $\gamma$ antagonist G3335, $* P<0.05$. (C) Chromatin immunoprecipitation showing ERR $\alpha$ bound to the UCP1 enhancer element in differentiated $9^{+4} \mathrm{fMSCs}$, but not to a region in the coding sequence of the same gene. (D) Reporter assay using this construct in Cos-1 cells. ERR $\alpha$ activates the enhancer, and its activity is further trans-activated by PGC1 $\alpha$ and repressed by RIP140. The ERR $\alpha$ inverse agonist XCT790 prevents this induction. (E) ERR $\alpha$ expression in $10^{+1}$ and $9^{+4}$ isolates before and after 21 days of adipogenic differentiation. mRNA expression was determined by qPCR. Error bars indicate SD. 
isolates, both before and after differentiation into adipocytes (Figure 2D), although interestingly, its expression was significantly higher in the $9^{+4}$ (brown) fMSCs than in the $10^{+1}$ (white) cells. It would therefore appear that certain isolates of fMSCs have a propensity to differentiate into white adipocytes and others into brown adipocytes, despite identical differentiation conditions. It is also noteworthy that basal UCP1 expression could be further induced by treating with isoproterenol to mimic the effects of $\beta$-adrenergic agents (Figure 2E). Thus, the $\mathrm{BM} 9^{+4}$ isolate retained properties expected in brown adipocytes. It should be noted that these two isolates came from fetuses differing by only 4 days in gestational age and were examined at similar passage number.

ERRa is a regulator of $U C P 1$ expression in human $f M$ $S C$-derived brown adipocytes

A number of nuclear receptors have been shown to regulate UCP-1 gene expression [10, 23]. Therefore, differentiated $9^{+4}$ cells were treated with agonists or antagonists for relevant nuclear receptors, and the effects on UCP1 mRNA levels were determined.

In keeping with the known role of PPAR $\gamma$ in regulating UCP1 expression, its agonist rosiglitazone resulted in a fourfold induction of UCP1 expression, whereas the antagonist G3335 resulted in a 50\% inhibition of UCP1 expression (Figure 4A and 4B). We postulated that the residual expression might reflect the action of other nuclear receptors. We found that an inverse agonist for ERRa, XCT790 [24], resulted in almost complete suppression of UCP1 expression (Figure 4A). Thus, we conclude that while PPAR $\gamma$ activation contributes to the expression of UCP1, ERR $\alpha$ is the predominant nuclear receptor responsible for its expression in the absence of $\beta$-adrenergic stimulation. Expression of the fatty acidbinding protein FABP4 was also examined. This is a well-characterized target of PPAR $\gamma$, and accordingly its expression was increased by $\sim 15$-fold following treatment with Rosiglitazone (Supplementary information, Figure S2). However, XCT790 did not reduce expression of this gene, and in fact resulted in a small induction. Thus, it appears that PPAR $\gamma$ and ERR $\alpha$ control transcription from distinct subsets of genes in these adipocytes.

Previous analysis of both the mouse and human UCP1 gene has identified an enhancer responsible for mediating the stimulatory effects of a number of nuclear receptors [23, 25], and several putative ERR $\alpha$-binding sites were identified (Supplementary information, Figure S3). We therefore performed chromatin immunoprecipitation experiments to investigate the binding of ERR $\alpha$ to the enhancer region of UCP1. We found that ERR $\alpha$ was bound in the vicinity of the UCP1 enhancer element in differentiated adipocytes from the $\mathrm{fMSC} 9^{+4}$ isolate, but not the last coding exon, which we tested as a negative control. To confirm that the effects of ERR $\alpha$ were mediated through this site, the 220-bp enhancer element of the human UCP1 gene was cloned into the reporter plasmid pGL3-TK and transiently transfected into Cos1 cells. We found that the expression of ERR $\alpha$ resulted in a $50 \%$ increase in reporter activity and this was further increased by the expression of PGC1 $\alpha$ (Figure 4D). Importantly, the ERR $\alpha$ inverse agonist XCT790, reduced the induction of reporter gene activity by ERR $\alpha$ and PGC1 $\alpha$. PGC1 $\alpha$ alone had no effect on activity. Conversely RIP140 expression blocked the ability of ERR $\alpha$ and PGC1 $\alpha$ to activate transcription, in keeping with its role as a suppressor of UCP1 transcription in murine adipose tissue.

ERR $\alpha$ mRNA levels were then determined in both the stem cells and adipocytes derived from the two isolates of fMSCs described above. ERR $\alpha$ expression was similar in the two isolates both before and after differentiation, although both lines expressed higher levels than adult sub-cutaneous adipocytes (Figure 4E). Thus, although ERR $\alpha$ is required for the expression of UCP- 1 in the $9^{+4}$ cells, it is not sufficient in the absence of other factors.

\section{Discussion}

Our study demonstrates the ability of fMSCs to differentiate into either brown or white adipocytes under identical cell culture conditions. Both regulators such as PPAR $\gamma$ and markers such as FABP4 were upregulated during differentiation in a similar manner to that seen in well-described murine and human adipocyte cell models $[2,20,26,27]$. Of note, the adipocyte-specific isotype of PPAR $\gamma$, PPAR $\gamma 2$, was markedly upregulated during adipogenesis in contrast to a previous report in which cells from second trimester amniotic fluid were used [17]. This difference might reflect the greater proportion of cells undergoing adipogenesis ( $>30 \%)$ in our study, although a fundamental difference between the first-trimester MSCs used here and second trimester cells cannot be ruled out. It should be noted that an even higher proportion of cells underwent adipogenic differentiation in previous studies that used single cell clones [18]. However, due to the limited survival of isolated MSCs in culture, we did not feel this would be a viable approach to proceed to fully characterize the differentiated adipocytes. Of particular interest is the pattern of expression of the preadipocyte marker Pref-1, which rises early in the differentiation process, prior to falling in the later stages. The induction of Pref-1 expression suggests that the stem cells first commit to the adipogenic lineage, acquiring the features 
of preadipocytes, prior to undergoing differentiation into mature adipocytes.

The ability of fMSCs to differentiate into brown adipocytes was dependent on the isolate tested and was unaffected by the precise hormonal treatments used to induce differentiation. Interestingly, the $\mathrm{BM}^{+4}$ isolate, which could undergo differentiation into brown adipocytes, was also able to differentiate into other mesenchymal lineages. The discovery of an isolate of fMSCs that differentiated into brown adipocytes suggests that this specialized adipose cell is indeed derived from the mesenchymal lineage, along with white adipocytes. This is in keeping with recent observations that brown adipocytes may share developmental origins with muscle cells, which are also of mesenchymal origin. A comparison of this isolate with $\mathrm{BM} 10^{+1} \mathrm{fMSC}$ that differentiates into white adipocytes shows that differences in expression of the nuclear receptor coregulators RIP140 or PGC1 coactivator cannot explain the alternative fates of the two isolates, despite the role for these regulators in mature white and brown adipocytes. Interestingly, PRDM16 was expressed at a higher level in the $9^{+4}$ isolate that was capable of differentiating into brown adipocytes, both before and after differentiation. Nevertheless, given that PRDM16 was also expressed in the $10^{+1}$ isolate, albeit at lower levels, it seems that PRDM16 is not sufficient to account for the alternative cell fates of these two isolates. It remains unclear whether these cells represent an in vivo source of both white and brown adipocytes and what factors may influence stem cell commitment during development.

A number of nuclear receptors have been shown to regulate Ucp 1 expression, including PPAR $\gamma$ and the retinoic acid receptor [23]. In this study, analysis of $\mathrm{UCP} 1$ expression in the $\mathrm{fMSC}$-derived brown adipocytes has shown a novel role for the orphan nuclear receptor ERR $\alpha$ in regulating human UCP1 expression. ERR $\alpha$ is expressed at high levels in brown fat, and is important in the regulation of many genes involved in fatty acid metabolism, such as medium-chain acyl coenzyme A dehydrogenase [28]. The role of this receptor in Ucp1 gene regulation in the mouse is unclear. Mice devoid of ERR $\alpha$ express increased amounts of Ucp 1 in their white adipose tissue [29], suggesting that ERR $\alpha$ or one of its downstream target genes may repress Ucp 1 expression in this tissue. However, more recent studies have not shown a role for this receptor in the regulation of Ucp1 in murine brown fat [30]. Nevertheless, it does regulate many genes important for mitochondrial function and fatty acid oxidation in brown adipocytes so that ERR $\alpha$-null animals have impaired adaptive thermogenesis. On the other hand, ERR $\alpha$ has been shown to be important for the increased expression of Ucp1 found in mouse white adipocytes lacking RIP140, and the elevation of Ucp1 in these cells is not dependent on adrenergic signaling [31]. Strikingly, brown adipocytes differentiated from fMSCs also express UCP1 in the absence of $\beta$-adrenergic signaling, and therefore ERR $\alpha$ activation may represent a common pathway for expression of this gene independently of cold stimulation. We have shown that ERR $\alpha$ activity is required for the expression of UCP1 in $\mathrm{MMSC}$-derived brown adipocytes, that ERR $\alpha$ can alter the transcriptional activity of the human UCP1 enhancer and that the receptor binds to the UCP1 enhancer in these cells.

Interestingly, the one physiological situation where brown fat is present and active prior to cold exposure is in the neonate, particularly the neonates of larger mammals, including humans, who are born with fully functional brown adipose tissue (reviewed in Cannon and Nedergaard [1]). This contrasts with mice, where brown adipose tissue is recruited in response to cold over the first few days of life. Thus, it is possible that these fMSCs are differentiating into adipocytes resembling the brown fat found in neonates, and may hint at a role for $\mathrm{ERR} \alpha$ in this neonatal period. Furthermore, the demonstration that ERR $\alpha$ can regulate human UCP1 expression in these cells suggests that increasing ERR $\alpha$ activity may be a viable therapeutic strategy to induce UCP1, and hence increase uncoupling as a potential therapy for obesity. Thus, along with giving insights into the developmental origin of human brown adipocytes, we have also demonstrated that these cells can be useful in elucidating signaling pathways using classical molecular biological techniques that have been difficult to apply to existing human models.

In conclusion, this study has demonstrated that fMSCs are a valid and viable model for studying aspects of adipogenesis that cannot be addressed with traditional models. There seems to be heterogeneity among adipocytes derived from different isolates of these cells, and this seems to be due to innate differences in the stem cells. The underlying mechanisms of these differences are unclear and further studies to determine this may be of importance in further understanding determinants of cell fate within the adipocyte lineage. Nevertheless, these studies have given insights not only into human brown adipogenesis but also revealed novel molecular pathways controlling the expression of key genes within brown adipocytes.

\section{Materials and Methods}

\section{Cell culture}

Human fMSCs were obtained from normal first-trimester hu- 
man fetuses at clinically indicated termination of pregnancy, as described elsewhere [18], after obtaining a written maternal consent and in accordance with institutional ethical approval and the national guidelines on the use of fetal tissue in research. Gestational age was determined by crown-rump length (CRL) on intraoperative ultrasound. Briefly, first-trimester FB samples (20-800 $\mu 1)$ were obtained by ultrasound-guided cardiac aspiration between 9 and 11 weeks of gestation prior to clinically indicated surgical termination of pregnancy. Heparinized (by flushing with heparin sodium 1000 units/ml from CP Pharmaceuticals), disposable siliconized 20-G 15-cm needles (COOK Ltd, UK) attached to 1-ml syringes were used for cardiocentesis. Fetal gestational age was determined by CRL measurement referenced to standard biometry charts (Robinson et al. [32]).

First-trimester fetal bone marrow tissues were collected after clinically indicated surgical termination of pregnancy. Fetal BMs were flushed from marrows of fetal long bones or spines, while FL was diced. Tissues were then washed with phosphate-buffered saline (PBS) and filtered through a $40-\mu \mathrm{m}$ cell strainer (BD Biosciences) to yield cell suspensions.

Harvested cell suspensions are centrifuged at $1500 \mathrm{rpm}$ (400 $\times g$ ) for $5 \mathrm{~min}$ and the cell pellet resuspended in high-glucose Dulbecco's modified Eagle's medium (DMEM; Sigma, UK) supplemented with 10\% fetal bovine serum (FBS; BioSera, Batch selected for maximal MSC growth), $2 \mathrm{mM}$ L-glutamine, $50 \mathrm{IU} / \mathrm{ml}$ penicillin and $50 \mu \mathrm{g} / \mathrm{ml}$ streptomycin (Invitrogen) (D10 medium). They were then plated in 100-mm tissue culture dishes (Corning) at a density of $10^{6}$ cells per $\mathrm{ml}$ and incubated at $37^{\circ} \mathrm{C}$ with $5 \%$ carbon dioxide. After 3 days, they were washed in PBS twice to remove non-adherent cells. Established adherent cell colonies were detached with $0.05 \%$ Trypsin-EDTA (StemCell Technologies) when they reached $70 \%-80 \%$ confluence and replated at $10^{4}$ cells per $\mathrm{cm}^{2}$ in $100-\mathrm{mm}$ dishes.

Cells derived from whole isolates of either fetal blood or bone marrow from single fetuses were expanded and passaged in DMEM with $10 \%$ FBS. Adipogenic differentiation was induced in early passage cells $(n=3-6)$ by plating the fMSC at $2 \times 10^{4}$ cells $\mathrm{cm}^{2}$, allowing the cells to reach confluence and then incubating for a further $48 \mathrm{~h}$. The media was then changed to DMEM supplemented with $10 \% \mathrm{FBS}$ as described above and the following hormones were added: insulin $(10 \mu \mathrm{g} / \mathrm{ml})$, dexamethasone $(1 \mu \mathrm{M})$, indomethacin $(200 \mu \mathrm{M})$ and isobutylmethylxanthine $(0.5 \mathrm{mM})$ [18]. Media were changed every 4 days and differentiation continued for 14-21 days.

Bone differentiation was induced, as previously described [18].

Human adult sub-cutaneous preadipocytes were obtained from Cambrex (UK) and differentiated according to the manufacturers instructions.

Oil Red O stain was used to confirm the presence of lipid in cells. Cells were washed with PBS, then fixed in $2 \%$ paraformaldehyde, $0.2 \%$ gluteraldehyde in PBS for $15 \mathrm{~min}$ and then rinsed with PBS. They were then stained with Oil Red O stain (made up in isopropanol) for $10 \mathrm{~min}$ and rinsed in $60 \%$ isopropanol followed by PBS (adapted from Ramirez-Zacarias et al. [33]). Lipid droplets stained red were visualized by light microscopy. Von Kossa staining was performed by fixing the cells in $10 \%$ formalin for $1 \mathrm{~h}$, washing and staining with $2 \%$ silver nitrate for $10 \mathrm{~min}$ in the dark and then washing and exposing to light.

Ligand experiments were performed by incubating differenti- ated cells in the media supplemented with $10 \%$ FBS and insulin 10 $\mu \mathrm{g}$ alone for $48 \mathrm{~h}$ prior to the addition of the indicated ligand for a further $48 \mathrm{~h}$. Rosiglitazone was obtained from Alexxis (UK) and XCT709 from Sigma.

\section{Immunocytochemistry}

Cells were grown to $60 \%$ confluence on glass cover slips, fixed in $4 \%$ paraformaldehyde in $125 \mathrm{mM}$ HEPES ( $\mathrm{pH} 7.6 ; 10$ min, $4{ }^{\circ} \mathrm{C}$ ), re-fixed in $8 \%$ paraformaldehyde in HEPES (50 min, $4{ }^{\circ} \mathrm{C}$ ) and then permeabilized in $0.5 \%$ Triton X-100 in PBS, with gentle shaking for $30 \mathrm{~min}$. Next, cells were blocked with PBS+ (PBS containing $1 \%$ BSA, $0.2 \%$ fish gelatin and $0.1 \%$ casein at $\mathrm{pH}$ 7.6) for $1 \mathrm{~h}$, incubated overnight $\left(4{ }^{\circ} \mathrm{C}\right)$ with the appropriate primary antibody in $\mathrm{PBS}+$, washed for $1.5 \mathrm{~h}$ in $\mathrm{PBS}+$, incubated with secondary antibodies in PBS+ for $1 \mathrm{~h}$, washed overnight (4 ${ }^{\circ} \mathrm{C}$ ) in PBS+, washed in PBS and then mounted in 4',6 diamidino2-phenylindole Vectashield mounting medium (Vector Laboratories). Slides were viewed under fluorescence microscopy (Zeiss Axioscope I microscope). Images were captured using a photonics digital camera (Hamamatsu) and processed by iPlab (Scanalytics) software. The following primary antibodies were used: rabbit polyclonal IgG laminin (Sigma), mouse monoclonal IgG SH2, SH3 (BD Biosciences), mouse monoclonal IgG CD14, CD34, CD45 and vimentin (Dako). Secondary antibodies for immunofluorescence were donkey anti-mouse IgG conjugated with FITC (Jackson ImmunoResearch Laboratories) and goat anti-rabbit IgG conjugated to Alexafluor 488 (Molecular Probes).

\section{Quantitative RT-PCR}

Total RNA from cells was extracted with TRIZOL reagent (Invitrogen) according to manufacturer's protocols. In total, $1 \mu \mathrm{g}$ of the resulting RNA was treated with DNAse (Sigma) and then reverse transcribed with MMLTV-RT (Sigma) using oligo-dT primers (Invitrogen). Quantitative real-time PCR was performed on an Opticon2 cycler (MJ research), using JumpStart Taq ready mix for quantitative PCR (Sigma). Gene expression was normalized to the house keeping gene L19 and fold differences were calculated by the $\Delta \mathrm{C}_{t}$ method [34]. Data are expressed in arbitrary units. Primer sequences are available on request.

\section{Reporter assays}

The 220-bp enhancer element of the human UCP-1 gene was amplified by PCR using the following primers: forward CAA TTG GTA CCG AAC TTG CTG CCA CTC CTT TG and reverse ATT GGC TCG AGT CTG GAT TCC AAG GAG CAG G. The product was ligated into the vector pGL3-TK.

Reporter assays were performed in Cos-1 cells, in 96-well plates in phenol red-free DMEM supplemented with 5\% dextrose charcoal stripped serum. Reporter construct $(20 \mathrm{ng})$ was transfected along with $5 \mathrm{ng}$ of an internal control plasmid expressing Renilla luciferase constitutively, together with the indicated amounts of expression plasmids. Total amount of DNA transfected was kept constant by using varying amounts of an empty pCI vector. Transfections were performed with FuGene6 (Roche) transfection reagent according to manufacturers instructions. Indicated ligands were added after $24 \mathrm{~h}$. Luciferase activity was assayed $48 \mathrm{~h}$ posttransfection with the LucLite reagent, according to manufacturers instructions, and the luminescence read on a Victor2 plate reader (Perkin Elmer). Activity was normalized to the Renilla activity. 


\section{Chromatin immunoprecipitation}

Cells were incubated in PBS with dimethyl adipimidate $(2.4$ $\mathrm{mg} / \mathrm{ml}$ ) for $30 \mathrm{~min}$ in the dark at room temperature. They were then fixed in $1 \%$ formaldehyde in DMEM for $15 \mathrm{~min}$ at $37^{\circ} \mathrm{C}$. Cross-linked cells were lysed, sonicated and immunoprecipitated with protein A/G PLUS-agarose (SC-2003; Santa Cruz Biotechnology, Santa Cruz, CA, USA) and antibodies against ERR $\alpha$ (LSA5402; Lifespan, Seattle, WA, USA) or control normal rabbit IgG (SC-2027; Santa Cruz Biotechnology). DNA fragments were purified with a QIAquick PCR purification kit (Qiagen, Valencia, CA, USA) and used as templates in PCRs. The primers used for the UCP1 enhancer were F: AAC TTG CTG CCA CTC CTT TG, R: TCT GAC AGG CTC TGG GAA GT and the last coding exon as a control F: GGG TGA AGC CTC ATC TCA AA, R: TCG TTT CAG TTG TTC AAA GCA.

\section{Statistics}

Data were analyzed with GraphPad Prism software (GraphPad software, California, USA). Comparisons were made with the oneway ANOVA (analysis of variance) with Tukey's post-hoc analysis.

\section{Acknowledgments}

This work was funded by a Wellcome Trust Training Fellowship to DM (074456). PW is supported by a Wellbeing of Women project grant and NMF acknowledges salary support from the UK National Institutes of Health Research Biomedical Research Centre funding scheme.

\section{References}

1 Cannon B, Nedergaard J. Brown adipose tissue: function and physiological significance. Physiol Rev 2004; 84:277-359.

2 Farmer SR. Transcriptional control of adipocyte formation. Cell Metab 2006; 4:263-273.

3 Smas CM, Sul HS. Pref-1, a protein containing EGF-like repeats, inhibits adipocyte differentiation. Cell 1993; 73:725734.

4 Lee K, Villena JA, Moon YS, et al. Inhibition of adipogenesis and development of glucose intolerance by soluble preadipocyte factor-1 (Pref-1). J Clin Invest 2003; 111:453-461.

5 Moon YS, Smas CM, Lee K, et al. Mice lacking paternally expressed Pref-1/Dlk1 display growth retardation and accelerated adiposity. Mol Cell Biol 2002; 22:5585-5592.

6 Timmons JA, Wennmalm K, Larsson O, et al. Myogenic gene expression signature establishes that brown and white adipocytes originate from distinct cell lineages. Proc Natl Acad Sci USA 2007; 104:4401-4406.

7 Crisan M, Casteilla L, Lehr L, et al. A reservoir of brown adipocyte progenitors in human skeletal muscle. Stem Cell 2008; 26:2425-2433.

8 Seale P, Bjork B, Yang W, et al. PRDM16 controls a brown fat/skeletal muscle switch. Nature 2008; 454:961-967.

9 Seale P, Kajimura S, Yang W, et al. Transcriptional control of brown fat determination by PRDM16. Cell Metab 2007; 6:3854.

10 Puigserver P, Wu Z, Park CW, et al. A cold-inducible coacti- vator of nuclear receptors linked to adaptive thermogenesis. Cell 1998; 92:829-839.

11 Leonardsson G, Steel JH, Christian M, et al. Nuclear receptor corepressor RIP140 regulates fat accumulation. Proc Natl Acad Sci USA 2004; 101:8437-8442.

12 Christian M, Kiskinis E, Debevec D, et al. RIP140-targeted repression of gene expression in adipocytes. Mol Cell Biol 2005; 25:9383-9391.

13 Morganstein DL, Christian M, Turner JJ, Parker MG, White R. Conditionally immortalized white preadipocytes: a novel adipocyte model. J Lipid Res 2008; 49:679-685.

14 Lelliott CJ, Medina-Gomez G, Petrovic N, et al. Ablation of PGC-1beta results in defective mitochondrial activity, thermogenesis, hepatic function, and cardiac performance. PLoS Biol 2006; 4:e369.

15 Pittenger MF, Mackay AM, Beck SC, et al. Multilineage potential of adult human mesenchymal stem cells. Science 1999; 284:143-147.

16 Mackay DL, Tesar PJ, Liang LN, Haynesworth SE. Characterizing medullary and human mesenchymal stem cell-derived adipocytes. J Cell Physiol 2006; 207:722-728.

17 Gemmis PD, Lapucci C, Bertelli M, et al. A real-time per approach to evaluate adipogenic potential of amniotic fluidderived human mesenchymal stem cells. Stem Cell Dev 2006; 15:719-728.

18 Campagnoli C, Roberts IAG, Kumar S, et al. Identification of mesenchymal stem/progenitor cells in human first-trimester fetal blood, liver, and bone marrow. Blood 2001; 98:23962402.

19 Ryden M, Dicker A, Gotherstrom C, et al. Functional characterization of human mesenchymal stem cell-derived adipocytes. Biochem Biophys Res Commun 2003; 311:391-397.

20 Tomlinson JJ, Boudreau A, Wu D, Atlas E, Hache RJG. Modulation of early human preadipocyte differentiation by glucocorticoids. Endocrinology 2006; 147:5284-5293.

21 Nakamura T, Shiojima S, Hirai Y, et al. Temporal gene expression changes during adipogenesis in human mesenchymal stem cells. Biochem Biophys Res Commun 2003; 303:306312 .

22 Janderova L, McNeil M, Murrell AN, Mynatt RL, Smith SR. Human mesenchymal stem cells as an in vitro model for human adipogenesis. Obes Res 2003; 11:65-74.

23 del Mar Gonzalez-Barroso M, Pecqueur C, Gelly C, et al. Transcriptional activation of the human ucp 1 gene in a rodent cell line. Synergism of retinoids, isoproterenol, and thiazolidinedione is mediated by a multipartite response element. $J$ Biol Chem 2000; 275:31722-31732.

24 Busch BB, Stevens WC, Martin R, et al. Identification of a selective inverse agonist for the orphan nuclear receptor estrogen-related receptor alpha. J Med Chem 2004; 47:55935596.

25 Kozak UC. An upstream enhancer regulating brown-fat-specific expression of the mitochondrial uncoupling protein gene. Mol Cell Biol 1994; 14:59-67.

26 Sekiya I, Larson BL, Vuoristo JT, Cui JG, Prockop DJ. Adipogenic differentiation of human adult stem cells from bone marrow stroma (MSCs). J Bone Miner Res 2004; 19:256-264.

27 Hung SC, Chang CF, Ma HL, Chen TH, Low-Tone HL. Gene expression profiles of early adipogenesis in human mesenchy- 
mal stem cells. Gene 2004; 340:141-150.

28 Sladek R, Bader JA, Giguere V. The orphan nuclear receptor estrogen-related receptor alpha is a transcriptional regulator of the human medium-chain acyl coenzyme A dehydrogenase gene. Mol Cell Biol 1997; 17:5400-5409.

29 Luo J, Sladek R, Carrier J, et al. Reduced fat mass in mice lacking orphan nuclear receptor estrogen-related receptor alpha. Mol Cell Biol 2003; 23:7947-7956.

30 Villena JA, Hock MB, Chang WY, et al. Orphan nuclear receptor estrogen-related receptor alpha is essential for adaptive thermogenesis. Proc Natl Acad Sci USA 2007; 104:14181423.

31 Debevec D, Christian M, Morganstein D, et al. Receptor interacting protein 140 regulates expression of uncoupling protein 1 in adipocytes through specific peroxisome proliferator activated receptor isoforms and estrogen-related receptor alpha. Mol Endocrinol 2007; 21:1581-1592.

32 Robinson HP, Fleming JE. A critical evaluation of sonar "crown-rump length" measurements. Br J Obstet Gynaecol 1975; 82:702-710.

33 Ramirez-Zacarias JL, Castro-Munozledo F, Kuri-Harcuch W. Quantitation of adipose conversion and triglycerides by staining intracytoplasmic lipids with oil red O. Histochem Cell Biol 1992; 97:493-497.

34 Bustin SA, Benes V, Nolan T, Pfaffl MW. Quantitative realtime RT-PCR - a perspective. J Mol Endocrinol 2005; 34:597601.

(Supplementary information is linked to the online version of the paper on the Cell Research website.) 